\title{
Belphégor
}

Littérature populaire et culture médiatique

15-1|2017

1936: les Jeux olympiques dans la presse internationale

\section{Entre compétition médiatique, idéologique et sportive : l'« événement » Berlin 1936 au sein de la presse romande}

\section{Gianni Haver and François Vallotton}

\section{OpenEdition}

\section{Journals}

Electronic version

URL: http://journals.openedition.org/belphegor/835

DOI: 10.4000/belphegor.835

ISSN: 1499-7185

Publisher

LPCM

Electronic reference

Gianni Haver and François Vallotton, « Entre compétition médiatique, idéologique et sportive :

l'« événement » Berlin 1936 au sein de la presse romande », Belphégor [Online], 15-1 | 2017, Online since 07 July 2017, connection on 21 April 2019. URL : http://journals.openedition.org/belphegor/835 ; DOI : 10.4000/belphegor.835

This text was automatically generated on 21 April 2019

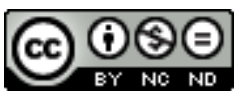

Belphégor est mis à disposition selon les termes de la Licence Creative Commons Attribution - Pas d'Utilisation Commerciale - Pas de Modification 4.0 International. 


\title{
Entre compétition médiatique, idéologique et sportive : l'« événement » Berlin 1936 au sein de la presse romande
}

\author{
Gianni Haver and François Vallotton
}

1 Les Jeux olympiques de Berlin en 1936 représentent à n'en pas douter l'un des événements phares de l'entre-deux-guerres et cela à plusieurs titres. Sur le plan de l'histoire de l'olympisme, ces manifestations quadriennales, dans la continuité d'un processus amorcé dès les Olympiades de l'après Première Guerre mondiale, accèdent progressivement au rang de rendez-vous au retentissement extra-européen, inscrit dans un temps et un espace renouvelés, tout en s'appuyant sur un rituel de plus en plus codifié et dont les enjeux débordent largement le cadre sportif. La médiatisation de l'événement s'amplifie et devient même le prétexte à l'expérimentation de nouvelles formes de retransmission et de communication ${ }^{1}$. Le cérémonial propre à la geste olympique se précise : le drapeau aux cinq anneaux fait son apparition à Anvers en 1920, la cérémonie de la flamme olympique est introduite à Amsterdam en 1928 alors que la remise des médailles avec podium et hymne national constitue la «marque de fabrique » de Los Angeles $^{2}$. Carl Diem, l'organisateur de la manifestation berlinoise, aura pour sa part l'idée du relais de la torche depuis Olympie. Enfin, l'élargissement des compétitions concernées, l'origine géographique de plus en plus large des athlètes mais aussi la présence croissante des femmes au sein des compétitions confèrent à ces manifestations une fonction de microcosme des rapports aussi bien politiques, idéologiques et sociaux des sociétés du temps.

2 Le sport, objet de prestige et de distinction, devient également une composante essentielle des relations internationales. Une section « Tourisme et Sport » est créée au sein du Service des Euvres françaises à l'étranger ${ }^{3}$ alors que les victoires de l'Italie lors des Coupes de monde de football de 1934 et 1938 sont autant de caisses de résonance pour la propagande mussolinienne. La participation même à l'olympiade berlinoise constitue 
un enjeu politique et diplomatique puisqu'un mouvement international de boycott est lancé via des associations juives et démocrates américaines.

3 Pour la Suisse, l'événement berlinois s'inscrit dans un contexte qu'il est important d'avoir à l'esprit pour mieux comprendre les enjeux des débats qu'il a nourris. Depuis 1935, le Conseil fédéral voit ses relations avec l'Allemagne parasitées par les suites de l'affaire Jacob, du nom d'un émigré israélite allemand enlevé à Bâle par la Gestapo. À cette crise diplomatique s'ajoutent deux épisodes qui mettent à l'épreuve la neutralité du pays et ses relations avec deux de ses puissants voisins. En octobre 1935, le conflit italo-éthiopien constitue une nouvelle épreuve pour la politique helvétique qui est confrontée à ses obligations en tant que membre de la Société des Nations et aux pressions des milieux économiques souhaitant maintenir leurs échanges avec le voisin du Sud. Par ailleurs, l'assassinat à Davos, début février 1936, du Gauleiter du parti national socialiste en Suisse par un étudiant en médecine de l'Université de Berne conduit Giuseppe Motta, le responsable du Département politique au sein de l'exécutif fédéral, à promettre à l'ambassadeur d'Allemagne un contrôle plus sévère des journaux suisses ${ }^{4}$.

D'autres enjeux sont liés à la situation du sport, ou plus exactement à la politique sportive helvétique. Au vu des efforts investis dans ce domaine par certains gouvernements, le volontarisme des autorités fédérales tend à apparaître, par contraste, encore embryonnaire. Qui plus est, des tensions se font jour dans l'espace public entre partisans d'un encouragement du sport de compétition, assujetti à la recherche de la performance, et les instances chargées de promouvoir l'éducation physique dans une optique essentiellement éducative et militaire ${ }^{5}$. Ces antagonismes sont redoublés par la présence sur le sol helvétique du mouvement olympique international : si plusieurs milieux voient d'un œil favorable les retombées positives de cette implantation - qui n'est par exemple pas étrangère au déroulement des Jeux de 1928 à Saint-Moritz -, d'autres, tout particulièrement en Suisse alémanique, dénoncent le poids croissant du Comité olympique national qui menace entre autres l'autonomie des grandes fédérations sportives ${ }^{6}$.

5 À un tout autre niveau enfin, la question du traitement des Jeux olympiques de Berlin renvoie aux transformations du paysage médiatique suisse dans ces années-là. Une intensification de la concurrence est engendrée d'une part par la diversification des supports (avec l'avènement d'une presse sportive spécialisée mais aussi l'essor de la presse illustrée), d'autre part par l'impact toujours plus grand du médium radiophonique. On assiste par ailleurs au développement d'interactions étroites entre le monde sportif et la sphère médiatique : le premier constitue une plus-value pour la presse en termes de publicité et d'attraction pour un large public, la seconde concourt à la popularité de certains sports et à l'élargissement des audiences lors d'événements et autres manifestations.

\section{Caractéristiques de la presse suisse francophone et constitution du corpus}

6 Jusqu'en 1982, le système médiatique suisse est caractérisé par une organisation très différenciée de la presse écrite et des médias électroniques ${ }^{7}$. À un système audiovisuel organisé autour de la création, dès 1931, d'une association disposant du monopole d'émission sur les ondes (la Société suisse de radiodiffusion) répond un système de presse 
dominé par des titres cantonaux et locaux liés aux principales agglomérations du pays. Ce paysage est caractérisé par une densité exceptionnelle, des tirages relativement modestes ${ }^{8}$, l'absence de journaux d'audience supracantonale jusque dans les années 1960 et la prédominance de cultures journalistiques très ancrées sur un terreau régional. Si les frontières entre journaux d'opinion et d'information sont parfois artificielles, on peut toutefois distinguer dans l'espace suisse romand des titres clairement reliés à des formations politiques' alors qu'une presse d'information bon marché (la Tribune de Genève en 1879 d'abord, suivie par la Tribune de Lausanne en 1893 et La Suisse en 1898) complète depuis la fin du XIXe siècle les feuilles d'avis traditionnelles beaucoup plus anciennes ( Feuille d'Avis de Lausanne, Feuille d'Avis de Neuchâtel). Parmi les transformations de l'entredeux-guerres, on soulignera l'émergence des premiers grands groupes de presse avec Lousonna en Suisse romande (Feuille d'Avis de Lausanne et Tribune de Lausanne) et Ringier, alors surtout actif dans le domaine de la presse illustrée en Suisse alémanique. Parallèlement, une segmentation de la production peut se lire dans l'avènement d'une presse sportive d'une part, l'essor d'une presse hebdomadaire illustrée d'autre part, qui se marque par la création du pendant romand de la Schweizer Illustrierte Zeitung - L'Illustré en 1921, et l'avènement de supports familiaux et féminins. Cette présence ascendante de l'image, encore timide dans la presse quotidienne, constitue une autre caractéristique de l'époque avec, dans la presse magazine illustrée, un progressif renversement de la subordination de la photographie par rapport au texte. Enfin, on remarque quelques tentatives d'imitation des hebdomadaires politico-culturels tels qu'ils fleurissent au même moment en France (Gringoire et Candide) : cette orientation est explicite dans l'hebdomadaire neuchâtelois Curieux, lancé l'année 1936, dont les positions idéologiques voisinent parfois avec celles de l'extrême-droite tout en défendant un projet journalistique ouvert à des rubriques très diversifiées.

7 Pour notre enquête sur le traitement des Jeux olympiques de Berlin dans la presse suisse francophone, nous avons privilégié différents types de supports. En premier lieu la presse quotidienne d'information dont une grande partie est désormais numérisée et donc consultable par mots-clés: il s'agit pour la presse lausannoise de la Feuille d'Avis de Lausanne, de la Tribune de Lausanne, de La Revue et de la Gazette de Lausanne, pour Genève du Journal de Genève, pour Neuchâtel de la Feuille d'Avis de Neuchâtel, pour La Chaux-deFonds de La Sentinelle et de L'Impartial, enfin pour le Valais du Nouvelliste et du Confédéré. Nous avons complété cet échantillonnage par deux titres genevois majeurs - la Tribune de Genève et La Suisse -, deux titres socialistes - Le Droit du Peuple et Le Travail -, ainsi que l'hebdomadaire Curieux déjà mentionné. En ce qui concerne la presse sportive, nous avons dépouillé Le Sport suisse (créé dès 1905), Pro Sport (1923) et Le Soir (1930) ${ }^{10}$. Quant à la presse illustrée, nous avons recouru aussi bien à la première génération de la presse illustrée (Patrie suisse, 1893 ; Lectures du foyer, 1912) qu'aux nouveaux hebdomadaires familiaux qui naissent dans les années 1920 (L'Abeille, 1921 ; L'Illustré, 1921 ; Le Radio, 1923 ; En famille, 1925 ou encore le catholique Echo illustré, 1930) ${ }^{11}$.

8 Le corpus est très éclaté, rapidement gigantesque, complexe de par la diversité et le type des informations délivrées - résultats, dépêches d'agence, photographie, reportage, éditorial. Aussi avons-nous renoncé à un traitement quantitatif pour une analyse plus qualitative. Celle-ci vise à rendre compte du croisement de regards très différenciés en fonction des orientations politiques des supports mais aussi de la nature, et donc des lectorats, de ceux-ci. Au-delà des résultats de la compétition, c'est bien tout ce que celle- 
ci met en jeu, sur un spectre thématique particulièrement large, qui nous intéresse ici, en sortant l'événement du cadre propagandiste étroit dans lequel on l'a souvent confiné.

\section{Les échos du mouvement de boycott en Suisse}

Les premières occurrences de l'événement berlinois interviennent dès 1935 et s'inscrivent dans un contexte lausannois bien spécifique. Depuis 1933, une Municipalité socialiste est aux commandes alors que la crise économique produit des effets de plus en plus dévastateurs en termes d'emplois et de conditions de vie. Elle y répondra par une politique sociale volontariste ainsi que la mise en œuvre de grands travaux qui passe par l'édification des bains publics de Bellerive au bord du lac. Imprégné des idées d'émancipation par le sport présentes au sein du mouvement sportif ouvrier organisé ${ }^{12}$, le syndic, Arthur Maret, propose même la candidature de la Ville pour l'organisation des Jeux olympiques de 1944. La Gazette de Lausanne, proche du parti libéral et de ce fait opposant résolu à la Municipalité en place, prend prétexte de la proximité de l'événement berlinois pour railler l'initiative socialiste : «Tenez-vous bien, sportifs! Le journal antipatriote de Nicole ${ }^{13}$ n'a, dirait-on, d'autre désir que celui de voir le drapeau helvétique flotter au sommet du mât olympique. [...] Le chancelier Hitler considère les prochains Jeux olympiques de Berlin comme une apothéose de la jeunesse nationaliste du monde; M. Maret, syndic de Lausanne, aurait-il le même idéal ? $^{14}$ ». Dans la suite de l'article, le journaliste prend ses distances, au nom du refus du sport-spectacle, avec une politique de grands travaux qui intégrait également la construction d'un stade municipal. Les hostilités sont ainsi ouvertes selon des lignes de position assez atypiques au demeurant.

Les fronts vont se renverser en juin 1935 lorsque, à la surprise générale et en dépit d'un intense travail de lobbying du Comité olympique suisse (COS), le Conseil national - l'une des deux Chambres du Parlement fédéral - refuse un crédit de 36.000 francs destiné à la préparation de la délégation suisse à Berlin. Ce sont les socialistes qui sont à l'origine du camouflet : ceux-ci dénoncent un geste aussi fort en période de restriction budgétaire et évoquent l'attitude peu respectueuse du voisin allemand dans l'affaire Jacob ainsi que la censure en Allemagne de plusieurs journaux alémaniques jugés trop critiques. Plusieurs députés bourgeois, aussi sensibles aux arguments financiers qu'ils sont peu convaincus de la nécessité d'une participation aux Jeux, contribuent par leur vote à mettre le gouvernement dans l'embarras. C'est en effet un séisme qui connaît rapidement des répliques en Allemagne. La presse bourgeoise réagit de manière virulente et fait front pour dénoncer une instrumentalisation malvenue de la gauche; quant au Ministre de Suisse à Berlin, Paul Dinichert, il écrit à Motta pour lui demander des explications. Dans la presse de gauche, la musique est différente et on se félicite de cette marque d'indépendance. L'affront sera toutefois vite réparé puisque la chambre des cantons désavoue le Conseil national qui, en deuxième lecture, entérine le crédit. Il aura toutefois fallu la remise au pas au sein de la droite des députés peu concernés en leur faisant valoir l'enjeu de la question pour les relations diplomatiques entre les deux pays et les retombées économiques et politiques du soutien au sport suisse ${ }^{15}$.

11 Loin de désarmer, la gauche va relayer de manière toujours plus assidue le mouvement de boycott des Jeux au niveau international. Le Travail comme SATUS Sport, l'organe de la Fédération ouvrière suisse de gymnastique et de sport, reprennent les mots d'ordre des deux grandes internationales sportives, l'ISOS plus réformiste, et l'IRS prosoviétique ${ }^{16}$. Comme le montre le travail de Christian Favre, la SATUS va s'impliquer tout 
particulièrement dans ce combat en dévoilant les persécutions dont sont victimes en Allemagne les opposants politiques mais aussi les juifs ${ }^{17}$. L'aveuglement du Comité olympique suisse mais aussi celui des principales fédérations sportives helvétiques sont également stigmatisés. Ce mouvement se poursuit durant la première moitié de l'année 1936 à l'occasion des Jeux de Garmisch tout d'abord puis lors de la mise sur pied du «Comité Fair Play pour le respect de l'esprit olympique » à Paris début juin. Même si cette dernière mobilisation ne peut plus remettre en question la tenue des Olympiades hitlériennes, elle a suffisamment d'impact pour susciter l'inquiétude du gouvernement allemand ainsi que des représentants du Reich en Suisse. Du côté de la SATUS, tous les efforts se reporteront désormais sur l'organisation des Olympiades populaires prévues à Barcelone en juillet : une délégation suisse de près de 300 sportifs socialistes est prête à partir depuis Genève avant que l'éclatement de la guerre civile ne débouche sur l'annulation de la manifestation. Berlin n'a plus de concurrente ; la presse socialiste suisse y répondra désormais dans sa grande majorité par le silence.

\section{La propagande allemande en Suisse}

Comme l'ont montré différents auteurs, les Jeux de Berlin se distinguent par leur gigantisme, mais aussi par une orchestration médiatique spectaculaire en amont de la manifestation. La propagande de presse commence dès 1932 puisque le premier collaborateur du Comité d'organisation a parmi ses tâches les relations avec les médias. Un service de presse olympique est mis sur pied dès 1933, propre à atteindre les agences, journaux et autres revues les plus importants dans le monde. Le Comité d'organisation fait par ailleurs paraître dès 1936 un luxueux mensuel en quatre langues, Olympische Spiele , qui s'adresse plus spécialement aux Comités des diverses organisations sportives de tous les pays susceptibles de se faire représenter aux Jeux. Cet effort contribue à ce que l'événement berlinois soit présent dans les colonnes des journaux suisses bien en amont de la manifestation elle-même ${ }^{18}$.

13 Le Docteur Francis-Marius Messerli, secrétaire du Comité olympique suisse, ne va pas ménager ses efforts pour promouvoir une image positive des Jeux dans l'espace public helvétique. Médecin, chargé d'enseignement au Cours supérieur d'hygiène à la Faculté de médecine de Paris, Messerli partage avec Coubertin la même foi dans les bienfaits de l'activité physique. Il sera aussi l'un des principaux pourfendeurs en Suisse du mouvement de boycott en affirmant la neutralité du sport et du CIO. En novembre 1935, il saisit le prétexte d'une séance du Comité olympique suisse à Zurich pour orchestrer, en liaison étroite avec l'ambassade d'Allemagne, une vaste opération de communication visant à rassurer sur la capacité des nazis à respecter l'esprit de la compétition olympique. Messerli peut compter sur la présence de Coubertin, annoncée par ce dernier comme sa dernière apparition en public: l'événement, radiodiffusé dans de très nombreux pays, réunit en outre quelques caciques du sport allemand, les représentants diplomatiques du Reich et plusieurs officiels suisses. Si l'écho semble avoir été moindre en Suisse francophone, ce n'est pas faute d'avoir choyé tout spécialement les représentants de la presse suisse, invités à un vol en avion la veille et conviés à une grande réception offerte par la Reichsbahnzentrale für den deutschen Reiseverkehr ${ }^{19}$.

14 Les Jeux d'hiver de Garmisch doivent être l'occasion pour l'Allemagne de lever les derniers doutes dans l'opinion. En Suisse, les positions sont contrastées en fonction des appartenances politiques. L'assassinat de Wilhelm Gustloff, le principal leader des 
organisations nazies en Suisse, le 4 février 1936, a sans doute pour effet de mettre sous le boisseau toute prise de distance critique dans la grande presse. Celle-ci est en effet rendue responsable d'un climat jugé anti-allemand par les représentants du régime. La raison d'Etat commande un profil bas ${ }^{20}$.

Quelques semaines plus tard, une série d'articles publiés dans l'hebdomadaire dominical Le Soir en avril 1936 revient sur la question des Jeux de Berlin en donnant la parole à certaines personnalités du monde sportif suisse. Les voix favorables au boycott y sont clairement minoritaires alors que plusieurs responsables de fédérations sportives plaident pour une nette séparation des enjeux politiques et olympiques. Outre l'inévitable Messerli, la voix de Marcel Suès, l'un des reporters suisses les plus populaires du moment de par son activité radiophonique, se démarque par son retour enthousiaste sur les Jeux de Garmisch. Ses inflexions se modulent toutefois de manière assez différente par rapport aux propos qu'il livrait au lendemain même des épreuves bavaroises: dans un article intitulé «Ce qu'il faut avoir le courage de révéler après les Jo d'hiver " ${ }^{21}$, il dénonçait en effet la déplorable organisation de la délégation suisse en pointant plus particulièrement du doigt les responsabilités du Docteur Messerli et du Comité olympique suisse. Au vu des résultats mitigés de l'équipe suisse, Suès ne sera pas le seul à décerner aux dirigeants du sport helvétique un brevet d'incapacité. Face à la détermination des partisans du boycott d'une part, le renforcement des critiques socialistes vis-à-vis de la remilitarisation de la Rhénanie, le bouillant reporter va atténuer ses critiques. Une attitude personnelle qui reflète une forme d'«union sacrée " qui s'opère dans les semaines qui précèdent l'inauguration berlinoise : au nom d'objectifs différents, le $\cos$, les fédérations sportives et les milieux politiques de droite uniront leurs efforts pour endiguer le mouvement de contestation. Un consensus qui reste néanmoins fragile et qui sera fortement mis à l'épreuve dès le début des compétitions.

\section{Une délégation de journalistes massive}

Selon une liste des accréditations retrouvées dans les archives $\mathrm{du} \mathrm{CIO}^{22}$, on peut établir que la délégation journalistique suisse à Berlin est parmi les plus nombreuses sur le plan international : on compte en effet 54 Suisses (16 Romands) représentant 32 titres. En guise de comparaison, on peut repérer 62 Français, 34 Belges et 7 Canadiens. Cette présence importante est évidemment à relier au caractère multilingue et à la diversité du paysage médiatique helvétique; y auront sans doute contribué aussi la proximité géographique, les contacts bilatéraux de toute nature avec l'Allemagne et l'intérêt engendré par l'événement depuis plusieurs mois. Au sein de la délégation, plus d'un journaliste sur deux est lié à une presse plus spécialement sportive : signe des moyens mis à disposition pour satisfaire la curiosité du public, un hebdomadaire comme Le sport suisse choisit de paraître deux fois par semaine durant la durée des Jeux. Pour la presse quotidienne, si plusieurs plumes témoignent de la place toujours croissante du sport dans l'économie du journal, d'autres sont manifestement là pour couvrir autant l'événement politique que sportif. Signalons encore que l'olympiade germanique marque l'importance de la radio comme relais de l'événement. Déjà présente à Garmisch, elle annonce, dans le radio-programme romand, une plage quotidienne vespérale d'une demi-heure consacrée à des reportages sur les compétitions les plus importantes ${ }^{23}$. Ceux-ci seront assumés par Maurice Aeschimann, le rédacteur de la Gazette de Lausanne. 
17 Sur le plan quantitatif, la part des pages sportives dans la grande presse, tout particulièrement celle d'information, est remarquable. Cette institutionnalisation de la rubrique sportive remonte au tournant du siècle, le quotidien genevois La Suisse étant considéré comme le journal précurseur dans ce domaine en Suisse romande ${ }^{24}$. La surface consacrée au sport dans ce journal avoisine les $4 \%$ en 1905, $11 \%$ en 1925 (un tiers lors de l'édition du lundi) et $9 \%$ en $1945^{25}$. L'actualité olympique sera l'occasion d'introduire, dans la plupart des titres romands, des rubriques quasi quotidiennes consacrées au rendez-vous berlinois, avec un apport assez systématique de la photographie. Un mémoire de master consacré au poids de la couverture des Jeux de 1936, 1968, 1984 dans la presse lausannoise souligne que, pour deux des trois journaux traités, les pourcentages sont supérieurs en 1936 qu'en $1968^{26}$. En termes de photographies, la Tribune de Lausanne (41) se démarque devant la Feuille d'Avis de Lausanne (30), la Gazette de Lausanne n'en offrant, comme pour toute autre actualité, aucune. Dans la presse quotidienne, les photographies ne portent nulle mention d'agence ou de photographe : selon un dispositif déjà en vigueur à Los Angeles et Garmisch, l'exclusivité des images de l'événement est confiée à des photographes nationaux, accrédités par le Comité d'organisation. Ce dispositif, présenté comme propre à éviter que les preneurs de vues se gênent les uns les autres, a aussi pour fonction de contrôler les représentations proposées ensuite aux rédactions internationales. Nous avons toutefois pu repérer dans notre dépouillement quelques images qui enfreignent manifestement ces dispositions : il s'agit d'un reportage de L. Jeck au quartier suisse du Village olympique publié dans L'Illustré2 ${ }^{27}$ ainsi qu'une photographie de la délégation suisse lors de la cérémonie d'ouverture prise de manière manifestement improvisée, du haut de la tribune ${ }^{28}$. La presse illustrée tente de parer à cette limitation en jouant sur une mise en page dynamique faisant alterner format horizontal avec images découpées ou en médaillons. Une autre spécificité de cette mise en récit par l'image est de faire dialoguer les échos des joutes berlinoises avec d'autres événements contemporains: une double page de L'Illustré oppose «en miroir» le reportage "La guerre fratricide» consacré à la guerre d'Espagne aux vues susmentionnées de la présence suisse au Village olympique ${ }^{29}$. Métaphoriquement, deux réalités s'affrontent : belliqueuse en Espagne, pacifique à Berlin.

18 À l'échelon romand, on peut observer que les principaux titres envoient des reporters sur place : la Gazette de Lausanne, Curieux, le Journal de Genève, La Suisse, la Tribune de Genève et la Tribune de Lausanne, certains journalistes travaillant pour plusieurs titres (Jean-Louis Clerc, par exemple, qui signe des articles pour Curieux et La Revue). Cette présence massive permet de compléter par une relation plus personnelle la partie rédactionnelle souvent importante consacrée aux résultats bruts ou à certaines dépêches. Si le dispositif mis en place par les organisateurs en matière de transmission des textes et des images autorise une information quasi instantanée, c'est bien le regard de « notre envoyé spécial » qui est susceptible d'apporter une couleur singulière et distinctive au traitement de cette actualité. Celui-ci s'attarde souvent sur les coulisses de l'événement ou sur des faits plus anecdotiques. C'est notamment le cas des reportages de Vico Rigassi ${ }^{30}$, l'une des voix de la radio romande qui est également le correspondant de la Tribune de Lausanne. Dès son arrivée, il souligne le respect à la lettre des consignes de politesse et de courtoisie transmises par Goebbels à la population et aux organisateurs; une visite au Village Olympique permet d'évoquer Luis Spiridon [sic] - le premier vainqueur du Marathon des Jeux modernes - déambulant en costume national, la nageuse américaine Eleanor Helm disqualifiée pour abus de champagne durant la traversée transatlantique ou encore la 
présence des athlètes «nègres $»^{31}$. Le spectacle est aussi dans les tribunes avec la mention des personnalités et autres têtes couronnées assistant à l'événement ${ }^{32}$. Dans le compte rendu sportif, l'habitude du micro déteint parfois sur le style journalistique; Rigassi s'efforce de donner la parole aux athlètes non sans se laisser aller parfois à des accents plus conformes aux marques de l'oralité : " "I bi z'friede" ["Je suis content", en dialecte alémanique], c'est par ces mots qu'Ernest Nievergelt nous a répondu lorsque nous lui avons serré la main. Le Zurichois, qui est un garçon charmant et fort bien élevé, nous a ensuite expliqué que tous les essais de démarrage durant l'épreuve ont été anéantis par la passivité des Allemands, des Belges et des Hollandais [...] "Nous avons fait tout ce que nous avons pu et je pense qu'on sera content de nous en Suisse". Certainement, brave Nievergelt, les sportifs suisses apprendront avec une joie sincère la nouvelle de votre superbe exploit ${ }^{33} »$. Rigassi incarne la figure du reporter sportif qui se veut proche des compétiteurs, de leurs émotions mais aussi de leurs attentes. Il souligne ainsi le contraste entre les récompenses offertes aux athlètes allemands victorieux et l'indifférence des autorités helvétiques qui n'ont même pas pris la peine d'un télégramme de félicitations à un marcheur méritant, par ailleurs au chômage. De la même manière, on observe une prise de distance récurrente vis-à-vis du gigantisme de compétitions qui s'éloignent aux yeux du reporter de l'esprit olympique souhaité par Coubertin ${ }^{34}$.

19 Comme le précise un long article dans Le Sport suisse, il y a deux types de reporters : les «fourmis » d'un côté, «qui se noient dans le détail, le nez fourré jour et nuit dans les dixièmes de seconde et les cheveux en quatre "; les "abeilles» de l'autre, "qui, en touristes, en amateurs, vont de stade en stade, de fleur en fleur, boire le meilleur $»^{35}$. Les premiers sont essentiellement présents dans les pages sportives, les seconds plus prompts à intervenir en première page ou dans les échos politiques. Pour ces derniers, le recul pris sur l'actualité immédiate peut aller jusqu'à une ironie parfois mordante, comme l'illustrent les interventions de Jean-Louis Clerc dans Curieux.

20 Au sein d'une presse quotidienne qui en est encore assez dépourvue, le recours fréquent aux photographies vient souligner l'exceptionnalité de l'événement. Dans la presse illustrée, les unes sont rares à l'exception d'une couverture de L'Echo illustré (l'arrivée de la torche olympique, 8 août) et de deux autres de L'Illustré (la victoire de l'étudiant japonais Son au marathon, le 13 août, et le retour de «nos" gymnastes, le 20 août): comme tend à le montrer ce dernier exemple, la pénurie d'images des athlètes suisses au sein de l'offre officielle explique sans doute ce nombre limité. Le recours à la photographie est aussi l'occasion d'un regard quelque peu distancié sur l'événement, au propre comme au figuré: des vues du Village olympique ou de lieux de compétition excentrés contrastent avec les reproductions récurrentes du stade olympique. Un illustré familial comme L'Abeille n'hésite pas par ailleurs à présenter certains points de vue décalés avec par exemple deux photographies consacrées respectivement au baiser d'un spectateur à un athlète ou aux embrassades d'une jeune plongeuse à son coach et mentor ${ }^{36}$. Cette présence plus importante que d'ordinaire des images dans la presse sportive et quotidienne est encore soulignée dans les colonnes du journal lausannois Pro Sport par le lancement d'un concours invitant les lecteurs à identifier, sur la base de productions partielles, certaines photographies de la manifestation olympique publiées dans ses colonnes $^{37}$. Sous une forme ludique, c'est une sorte de pédagogie de la lecture de l'image qui est ici proposée. 


\section{La multiplicité des regards sur l'événement}

21 Comme on l'a vu dans les mois qui précèdent l'inauguration des Jeux d'été, la polarisation est très forte entre presse bourgeoise et presse de gauche quant à l'interprétation de l'événement. Aux premiers commentaires louangeurs dans la presse bourgeoise concernant l'organisation et les infrastructures répondent les critiques socialistes, plus spécialement dans la presse alémanique. Celle-ci dénonce notamment la multiplication des réceptions réservées aux journalistes afin de tenter de les amadouer et de recueillir les échos les plus favorables ${ }^{38}$. Côté romand, Le Travail tout comme La Sentinelle vont s'abstenir progressivement de tout commentaire alors que Le Droit du Peuple, après avoir relayé fin juillet le contenu d'un tract berlinois condamnant la répression du régime, se limite à certains résultats.

$\mathrm{Au}$ sein de la presse de droite, des inflexions spécifiques peuvent toutefois être observées en fonction des titres. Ainsi, le Journal de Genève, proche du parti libéral et de la banque privée genevoise, garde durant l'ensemble des Jeux une attitude enthousiaste. Le Village olympique est notamment caractérisé comme « une petite république modèle, une société des nations du sport et de la jeunesse $\Perp^{39}$; en septembre, on souligne le succès des Jeux en relayant longuement le discours de Hitler au Congrès de Nuremberg: "Il ne sera plus possible désormais aux chroniqueurs juifs d'altérer la vérité et d'affirmer le contraire ${ }^{40}$. Son pendant idéologique lausannois, la Gazette de Lausanne, développe un jugement plus contrasté par l'entremise de son correspondant (politique) Camille Loutre et de son rédacteur Maurice Aeschimann. Dès la cérémonie d'ouverture, ce dernier souligne combien l'événement est encadré et pris en charge par les cadres du parti nazi : on évoque la présence des jeunesses hitlériennes, le discours de Goebbels et les chants patriotiques qui ponctuent la cérémonie du matin. De manière récurrente s'exprime un certain scepticisme quant au rôle pacificateur des Jeux. Dans un article « bilan » sur « Les enseignements des Jeux olympiques ", Aeschimann insistera ainsi sur la dégradation de l'idéal olympique: «Les Jeux olympiques, ce n'est même plus une lutte de nations, c'est un concours de races. Ce nouvel idéal est respectable. Nous préférions cependant l'ancien $»^{41}$.

23 Des voix plus critiques s'expriment de manière plus tranchée dans la presse radicale. En Valais, Le Confédéré stigmatise « un pays aveuglé par le chauvinisme et qui entend, par tous les moyens imaginables, prouver la suprématie de la race aryenne $!^{42}$ ». La Revue, organe du parti radical vaudois, joue pour sa part sur une certaine ambiguïté. Ainsi un article du 24 juin 1936, qui évoque notamment la manipulation à l'origine de l'incendie du Reichstag, est ponctué d'une prise de distance de la rédaction : "Nous laissons à notre collaborateur la responsabilité des déductions qu'il tire de certains ouvrages écrits par des émigrés allemands. Nous ne pensons pas notamment que le péril communiste ait été aussi imaginaire qu'on le dit ci-haut et nous ne croyons pas à l'effet décisif de "révélations" toujours discutables sur l'existence du régime $»^{43}$. Il n'en reste pas moins que le journal sera l'un des rares à soulever le problème des athlètes juifs en insistant sur l'hypocrisie des garanties nazies quant à leur égalité de traitement ${ }^{44}$. La Revue a également la particularité de pouvoir compter sur la collaboration du journaliste Jean-Louis Clerc qui livre parallèlement plusieurs papiers à l'hebdomadaire Curieux, pourtant bien plus marqué à droite sur l'échiquier politique. La plume de Jean-Louis Clerc se démarque surtout par sa tonalité volontiers ironique, voire irrespectueuse, tant vis-à-vis de certains 
représentants du comité d'organisation allemand que de certains officiels composant la délégation helvétique, Messerli en tête.

Les récriminations contre les Jeux vont s'intensifier dès lors que les résultats des athlètes suisses ne sont pas à la hauteur des attentes. Alors que les premières contre performances sont encore mises sur le compte d'une organisation du sport suisse déficiente, la disqualification d'un yachtman genevois pour professionnalisme d'une part, la désillusion de l'équipe suisse de gymnastique suite à des décisions de juges contestées d'autre part, intensifient une forme d'amertume face à l'instrumentalisation de l'événement par les nazis: "Tout en avouant notre incompétence sportive, nous constatons que de gigantesques exhibitions de cirque comme ces Jeux olympiques versent trop dans le nationalisme et qu'une compétition entre athlètes internationaux, c'est-à-dire surtout entre individus, ne saurait dégénérer en classements entre communautés nationales. [...] Il y aurait aussi à dire plus d'un mot sur les limites incertaines de l'amateurisme et du professionnalisme ${ }^{45}$ ». Ici aussi, Le Confédéré valaisan fait partie des plus virulents : « Ainsi, la Germanie triomphera. La moutonnaille aryenne bêlera d'admiration aux pieds du toutpuissant Hitler ! [...] Eh oui ! les Allemands ont été vexés de voir le peu d'enthousiasme que nous inspirent leurs méthodes toutes de force et d'oppression. On ne peut tolérer, à Berlin, que certains Suisses romands (parfaits ignorants de la langue de Goethe) n'aient pas écouté, 80 minutes durant, le discours du Fuhrer, chapeau bas, oreilles tendues et bouche close... Un Genevois aurait eu l'outrecuidance d'en "griller une » pendant le long exposé du chef nazi !6 $^{4}$ Le journal ira même jusqu'à saluer la décision du Pérou d'avoir quitté les Jeux suite à l'annulation de sa victoire en football contre l'Autriche...

Indépendamment des considérations sur le régime et l'instrumentalisation des Jeux à son profit, deux thématiques apparaissent avec récurrence dans les comptes rendus de la manifestation berlinoise. La première est liée à une forme de mondialisation sportive qui est rendue explicite par la participation de délégations asiatiques importantes ainsi que par la présence des athlètes noirs. À cet égard, si les performances d'Owens et de certains de ses compatriotes sont saluées avec admiration, on peut aussi remarquer le maniement de stéréotypes particulièrement dépréciatifs. Dans un article de la Gazette de Lausanne, l'image d'Owens n'a pas grand-chose à voir avec celle, rétrospective, de dieu du stade : « Il est marié depuis peu, était considéré comme professionnel il y a quelques mois, il se mouche dans ses doigts, mâche son chewing gum, et fait le désespoir de son entraîneur, M. Robertson. Owens, garçon fantasque, court et saute mieux que personne au monde. Mais ne lui demandez pas autre chose ${ }^{47} »$. Une série de photographies publiée dans la Tribune de Lausanne légende celles-ci «le péril noir» en signalant la supériorité des athlètes de couleur en athlétisme. Une disposition qui est à mettre en relation avec une série, publiée le lendemain et selon la même construction, présentant des portraits de coureurs blancs cette fois, avec comme légende «c'est du Nord aujourd'hui que nous viennent les triomphateurs ». L'athlète suisse Schwab, deuxième du 50 kilomètres, est associé à la galerie des vainqueurs nordiques, reliant de manière implicite l'homo helveticus à une forme de « résistance aryenne ».

Depuis plusieurs Olympiades, la présence toujours plus importante des femmes constitue une autre caractéristique du regard médiatique sur ces joutes. Comme l'ont montré différents travaux ${ }^{48}$, cette féminisation du corps sportif va se décliner selon des modalités multiples, de la «star" sportive incarnée par la patineuse - et future vedette cinématographique - Sonja Henie aux représentations des nageuses dont les poses glamour investissent régulièrement dès 1932 les magazines illustrés. Même cantonnée au 
registre du charme et de la séduction, cette présence féminine est loin d'être unanimement saluée, comme peut l'illustrer une contribution de Jean Peitrequin dans la Tribune de Lausanne: «[...] Des femmes qui lancent le javelot, qui jettent le disque, qui s'évertuent à triompher dans les cent mètres plats, quel intérêt voulez-vous que cela présente ? Toutes, ou presque toutes, paient leur couronne de chêne ou leur médaille de bronze du prix de leur féminité... ${ }^{49} »$. Aux yeux de celui qui n'est alors que conseiller communal et député radical (il deviendra syndic de la Ville de Lausanne en 1950), la manifestation olympique menace d'instaurer un nouvel ordre "genré » qui semble a priori plus menaçant que les enjeux idéologiques et raciaux soulignés par d'autres commentateurs.

\section{L'affirmation de valeurs nationales par le sport}

Dès le mois d'août, la piètre prestation des athlètes suisses va alimenter un débat sur la politique sportive suisse. Le secrétaire du Comité national olympique avait fanfaronné en promettant une moisson de médailles; la presse déchante. Le Soir évoque la « déchéance de nos sports nationaux ${ }^{50}$ » alors que d'autres titres ironisent sur la composition d'une délégation helvétique composée d'un grand nombre d'officiels dont l'utilité est fortement remise en question.

Par contraste, on ne manque pas de souligner l'exemple que constituent les modèles allemand et italien pour regagner une crédibilité sur la scène sportive, mais aussi politique, internationale. Pour le conseiller national vaudois Henry Cottier, une réforme fondamentale est urgente : «Il faut instituer une dictature du sport en Suisse. Pas celle, certainement, du cos dont les droits sont à revoir, mais celle d'un triumvirat, par exemple, qui réponde à nos principes fédéralistes. Ce triumvirat, que j'appellerai notre commissariat des sports - qui pourrait prendre exemple sur le Dopolavoro d'Italie aurait toutes compétences. Il aurait en mains toute l'organisation, la préparation et l'entraînement du sport en Suisse ${ }^{51}$ ». Dans un autre registre, certains milieux en appellent plus généralement à une forme de régénération des institutions helvétiques en établissant un parallèle entre l'organisation du sport en Suisse et un régime politique, miné par les combines politiciennes et considéré sur le déclin ${ }^{52}$.

Si une étatisation du sport est loin de faire l'unanimité, plusieurs voix se retrouvent pour souligner l'importance des activités physiques dans la formation d'une jeunesse saine mais aussi prête au combat : "L'État! L'État ! Est-ce bien notre rôle d'appeler l'Etat à la rescousse? Il y a dix ans, nous n'aurions pas tenu le même langage. Nous pensions alors que le sport pouvait s'épanouir librement, loin des lois et des uniformes. Mais il a failli à sa mission. [...] Il n'a réussi qu'à créer des cabotins ; une petite minorité de jeunes gens a usé et abusé du sport, tandis que la masse restait amorphe. [...] Songez que la jeunesse allemande qui cueille aujourd'hui des lauriers sur le stade peut être prête, demain, à faire une autre moisson. Que feront contre elle tant d'épaules étroites, tant de jambes grêles de chez nous? $\|^{53}$. Dans cette perspective, une structure centralisée avec un pilotage plus strict du Département militaire fédéral est l'objet des vœux de certains. Un rapport de la Commission fédérale de gymnastique et du sport propose en septembre 1936 une forme de bilan critique visant à réformer de fond en comble la politique sportive en Suisse ${ }^{54}$. Cela passe à ses yeux par le développement des activités physiques dans la population (avec notamment un enseignement de la gymnastique obligatoire jusqu'à l'âge de 20 ans) et la mise en place d'un meilleur encadrement des athlètes de pointe. Un mois plus tard, 
ce débat resurgit lors d'une séance de crise convoquée par la même organisation. Elle est l'occasion pour son président de prendre ses distances vis-à-vis d'une orientation unilatérale de la politique sportive sur les compétitions internationales. Au final, ces débats ne déboucheront sur aucune réforme d'importance. Il faudra attendre la guerre et un projet de loi sur la formation prémilitaire obligatoire pour que ces questions resurgissent dans l'espace public. En dépit d'un soutien très large des principales institutions sportives et au sein la classe politique, le projet est rejeté en votation populaire le $1^{\text {er }}$ décembre 1940 . Le retrait de prérogatives cantonales en matière d'éducation explique sans doute la netteté du vote ; pour le journal socialiste neuchâtelois La Sentinelle, le peuple a aussi manifesté son opposition à la volonté des autorités "d'imiter les régimes totalitaires dans leur emprise sur la jeunesse ${ }^{55}$ ». Cet épisode vient clore au demeurant les velléités de réforme directement suscitées par les Jeux berlinois.

30 Au final, cette enquête sur le traitement des Jeux olympiques de 1936 aura montré le poids de cet événement dans les médias mais plus largement dans la société suisse, et cela bien au-delà de la seule actualité du mois d'août 1936. Alors que les sujets communs sont rares dans une presse marquée par sa dimension régionale, voire locale, Berlin constitue un point d'intérêt très largement investi par les journaux suisses, dans leur hétérogénéité géographique et typologique. À l'exception notable toutefois du silence révélateur de la presse de gauche.

31 Autre élément remarquable, le très grand nombre d'envoyés spéciaux qui va autoriser un traitement plus personnalisé de l'événement: même si la plupart des rédacteurs ont intégré ce qu'il est possible de dire sur un enjeu d'actualité aussi brûlant et délicat politiquement, le sport est une manière de parler de politique de manière plus ou moins codée. Dans la concurrence exacerbée que connaît le paysage médiatique, les échos berlinois sont l'occasion d'affirmer une tonalité spécifique, aux inflexions somme toute plus diversifiées qu'attendues. Bien souvent en effet, l'analyse rétrospective de la couverture d'un événement politique dans la presse s'avère sans grande surprise: l'historien ne fait ainsi que constater le renforcement de clivages préexistants, entre presse d'opinion et d'information d'une part, organes d'orientation idéologique divergente d'autre part. De par le traitement concurrentiel de la même matrice informationnelle, des positionnements mais aussi des formes de narration propres se font jour dans la presse suisse qui agrègent de manière souvent insolite la logique du reportage, le compte rendu sportif et la vogue d'un journalisme mondain dont les athlètes sont les nouveaux protagonistes.

\section{NOTES}

1. L'événement berlinois fera l'objet de l'une des premières couvertures en direct de l'histoire de la télévision. Plus de 150000 personnes ont pu suivre les compétitions depuis certaines Bierstuben et autres auditoires à Berlin, Postdam et Leipzig.

2. Robert Frank, «Internationalisation du sport et diplomatie sportive», in Robert Frank (dir.), Pour l'histoire des relations internationales, Paris : PUF, 2012. 
3. Pierre Arnaud, «Sport et olympisme après la Première Guerre mondiale. Nouvelle donne géopolitique et enjeux de prestige», Relations internationales, $\mathrm{n}^{\circ}$ 111, automne 2002, pp. 347-363.

4. Sur ces épisodes, voir notamment Roland Ruffieux, La Suisse de l'entre-deux-guerres, Lausanne : Payot, 1974.

5. On soulignera le rôle joué en Suisse par l'Association nationale d'éducation physique (ANEP) fondée en 1917 qui devient dans les années 1920 la troisième instance dirigeante du sport helvétique aux côtés de la Commission fédérale de gymnastique de sport (CFGS) et du Comité olympique suisse (COS). Voir aussi Marco Marcacci, «Institutionnalisation et "militarisation" du sport en Suisse (1914-1945», in Sports en formes. Acteurs, contextes et dynamiques d'institutionnalisation, sous la dir. de Christophe Jaccoud et Thomas Busset, Lausanne : Antipodes, 2001, pp. 35-50.

6. Christian Favre, La Suisse face aux Jeux olympiques de Berlin 1936. Un pays partagé entre la contestation et la sauvegarde de ses intérêts avec le IIIe Reich, Université de Fribourg, 2004.

7. Ernest Bollinger, La presse suisse : structure et diversité, Berne : H. Lang ; Francfort/M : P. Lang, 1976; voir aussi Ulrich Saxer, «Culture médiatique», in Paul Hugger (dir.), Les Suisses. Modes de vie, Traditions, Mentalités, tome III, Lausanne, Payot, 1992, 1403-1431.

8. Selon Karl Weber (Tableau de la presse suisse, Berne: H. Lang, 1948), sur les 400 journaux recensés en Suisse, une petite dizaine atteint les 50000 exemplaires, une vingtaine entre 10000 et 50000 .

9. Dans notre corpus, la Gazette de Lausanne et le Journal de Genève pour le parti libéralconservateur, La Revue à Lausanne et Le Confédéré à Martigny pour le parti radical lié aux pères fondateurs de l'Etat fédéral, Le Nouvelliste en Valais pour les catholiques conservateurs, enfin La Sentinelle à La Chaux-de-Fonds, Le Travail à Genève et le Droit du peuple à Lausanne pour la presse socialiste.

10. Voir sur ce point Karim di Matteo, Les Débuts de la presse sportive suisse: son implication sociale, politique et économique pour le sport : l'exemple du Sport suisse (1905-1947), Mémoire en histoire sous la dir. de Sébastien Guex, Université de Lausanne, 2001.

11. Pour un aperçu, Gianni Haver, «La presse illustrée en Suisse, 1893-1945», in Gianni Haver (dir.), Photo de presse, Lausanne : Antipodes, 2009, pp. 39-65.

12. Voir Marco Marcacci, «Il était une fois le sport ouvrier: l'évolution du SATUS depuis la deuxième guerre mondiale», Cahiers d'histoire du mouvement ouvrier, № 19 : Dossier Sport ouvrier, 2002, pp. 11-21.

13. Léon Nicole (1887-1965) est l'une des principales figures du mouvement ouvrier en Suisse romande. Rédacteur du Travail, il est député (dès 1919) et conseiller national (dès 1919 également) socialiste genevois, élu à l'exécutif cantonal genevois en 1933.

14. Ae [Maurice Aeschimann], "A propos des Jeux olympiques. Le Droit du peuple et le sport», Gazette de Lausanne, 19 février 1935, p. 3

15. Sur ce débat, voir Christian Favre, La Suisse face aux Jeux olympiques de Berlin 1936, op. cit., p. 36 et ss.. Voir aussi Etienne Steiner, Les Lausannois et les Jeux olympiques de Berlin 1936, Mémoire en histoire sous la dir. de Pierre du Bois, Université de Lausanne, 1984.

16. Le mouvement international sportif ouvrier est divisé depuis le début des années 1920 entre une aile procommuniste (l'IRS, l'Internationale sportive rouge) et une aile réformiste (l'ISOS, l'Internationale sportive ouvrière socialiste).

17. Christian Favre, La Suisse face aux Jeux olympiques de Berlin 1936, op. cit., pp. 65-87.

18. Voir notamment A.Woelfli, «Les Jeux olympiques de 1936», La Revue, 7 novembre 1935, p. 3. Le Journal de Genève inaugure une chronique intitulée « Petites nouvelles des Jeux olympiques» le 31 décembre 1935.

19. Christian Favre, La Suisse face aux Jeux olympiques de Berlin 1936, op. cit., pp. 113-117.

20. Matthieu Gillabert, La propagande nazie en Suisse. L'affaire Gustloff, 1936, Lausanne : PPUR, 2008 (Le Savoir suisse ; 49). 
21. Me M.-W. Suès, «Ce qu'il faut avoir le courage de révéler après les JO d'hiver», Le Soir, 23 février 1936.

22. «Accréditations pour les Jeux olympiques d'été de Berlin 1936 : listes des journaux représentés et des journalistes, formulaires relatifs aux cartes d'identité et à la participation des officiels et carte d'identité»; Musée olympique (Lausanne), CIO JO-1936S-ACCRE, dossier 206705.

23. Le Radio, № 694, semaine du 26 juillet au $1^{\mathrm{er}}$ août 1936.

24. Karim di Matteo, Les débuts de la presse sportive suisse, op. cit., p. 33.

25. Ibid., p. 36.

26. La comparaison n'est pas forcément très pertinente au vu du caractère assez différent des deux événements et de l'éloignement géographique de Mexico. La part constituée par l'actualité olympique en 1936 par rapport à l'ensemble de la surface rédactionnelle est de 3,97\% pour la Feuille d'Avis de Lausanne, 4,96\% pour la Gazette de Lausanne et 8,12\% pour la Tribune de Lausanne; chiffres tirés de Julien Hostettler, L'évolution du grand événement sportif dans la presse lausannoise: Les Jeux olympiques de 1936, 1968 et 1984, Mémoire en histoire sous la dir. du Prof. François Jequier, Université de Lausanne, août 2004, pp. 32-33.

27. «Aux Jeux olympiques de Berlin», L'Illustré, № 33, 1936.

28. Ibid., № 32, 1936.

29. Ibid., № 33, 1936.

30. Vico Rigassi (1905-1983) est l'une des figures du journalisme sportif suisse les plus connues de par sa capacité à commenter des compétitions à la radio dans les trois principales langues nationales; il a collaboré à de nombreux journaux et revues en Suisse et à l'étranger.

31. V. R. [Vico Rigassi], «La fiévreuse attente à la veille des Jeux olympiques», Tribune de Lausanne, 30 juillet 1936, pp. 3-4.

32. V. R. [Vico Rigassi], «En marge des jeux», Tribune de Lausanne, 9 août 1936, p. 4.

33. V. R. [Vico Rigassi], «Aux Jeux olympiques. La belle course des cyclistes suisses», Tribune de Lausanne, 12 août 1936, p. 4.

34. «Nous avons derrière nous la laborieuse journée de l'inauguration solennelle et grandiose des Jeux. Cette manifestation sportive-militaire laissera à tous un souvenir inoubliable, car elle a été organisée de main de maître. Mais cette organisation contrastait avec l'esprit que le baron de Coubertin avait voulu donner aux Jeux olympiques»; V.R., «Impressions dominicales», Tribune de Lausanne, 4 août 1936, p. 5. Dans un autre article (17 août 1936), Rigassi parle de la fin de l'amateurisme tel que l'avait conçu le rénovateur des Jeux modernes.

35. Emile Birbaum, «Après les Jeux olympiques», Le Sport suisse, 22 août 1936.

36. L'Abeille, 29 août 1936.

37. Pro Sport, 17 août 1936.

38. Les titres les plus impliquées dans cette dénonciation sont le Berner Tagwacht, la Basler Arbeiter Zeitung et SATUS Sport ; voir Christian Favre, op. cit, pp. 167 et ss.

39. s.n., «Le cadre des Jeux olympiques», Journal de Genève, 31 juillet 1936, p. 1.

40. s.n., «La proclamation du Führer au congrès de Nuremberg», Journal de Genève, 10 septembre 1936, p. 2.

41. Ae [Maurice Aeschimann], «Les enseignements des Jeux olympiques I», Gazette de Lausanne, 24 août 1936, p. 1.

42. s.n., «Les Jeux olympiques de Berlin. L'Hitlérie et ses injustices», Le Confédéré, 14 août 1936, p. 3.

43. «L'Allemagne pacifique et muette (d'un correspondant particulier)», La Revue, 24 juin 1936, p. 1.

44. Noël Hiller, «L'histoire des Olympiades. Indiscrétions sur l'Olympiade de Berlin», La Revue, 28 juillet 1936, p. 1.

45. Camille Loutre, «Lettre de Berlin. Impressions d'olympiades», Gazette de Lausanne, 14 août 1936, p. 1. 
46. R. B., «En marge des Jeux olympiques. Pourquoi la Suisse a t'elle été battue ?», Le Confédéré, 16 août 1936, p. 3.

47. Ae, «Les XIe Jeux olympiques. Athlètes à vos marques !», Gazette de Lausanne, 3 août 1936, p. 3.

48. Voir notamment La mise en scène du corps sportif, Laurent Guido et alii (dir.), Lausanne : Musée olympique, 2002.

49. Jean Peitrequin, «Les femmes athlètes», Tribune de Lausanne, 16 août 1936, p. 3.

50. Le Soir, 22-23 août 1936.

51. s.n., «Une rénovation catégorique du sport en Suisse», La Revue, 18 août 1936, p. 3. On rappellera que le Dopolavoro avait été créé en 1925 par le régime mussolinien afin de prendre en charge et d'encadrer le temps libre des travailleurs.

52. Jean-Louis Clerc, «Après Berlin. La politique de l'olympiade», Curieux, 29 août 1936.

53. Ae [Maurice Aeschimann], «Les enseignements des Jeux olympiques. IV (1). Changeons de méthode», Gazette de Lausanne, 4 septembre 1936, p. 3.

54. Sur ces débats, voir Christian Favre, op. cit, p. 177 et ss.

55. La Sentinelle, 2 décembre 1940, cité par Favre, Ibid., p. 211. Sur la votation de 1940, voir aussi Marco Marcacci, «Institutionnalisation et “militarisation” du sport en Suisse (1914-1945)», op. cit..

\section{ABSTRACTS}

Les Jeux olympiques de Berlin constituent l'un des événements médiatiques les plus importants de l'entre-deux-guerres. Toutefois, si sa mise en scène par l'appareil étatique nazi a largement été explorée, les déclinaisons journalistiques auxquels ils ont donné lieu ont moins retenu l'attention. Au-delà de la prudence de mise pour ne pas froisser le puissant voisin, les échos de la manifestation en Suisse romande sont multiples, témoignant d'enjeux médiatiques, sportifs, politiques et sociaux que cette contribution se propose de décrypter.

\section{INDEX}

Mots-clés: jeux olympiques, 1936, médias, presse romande, propagande nazie, Suisse

\section{AUTHORS}

\section{GIANNI HAVER}

Gianni Haver est professeur associé de sociologie de l'image et d'histoire sociale des médias à l'Université de Lausanne. Il s'intéresse particulièrement aux productions médiatiques de la période de l'entre-deux-guerres et de la Deuxième Guerre mondiale et aux rapports entre histoire et médias. Il a notamment travaillé sur le cinéma et, plus récemment, sur les comics et la photo de presse. Il dirige la collection « Médias et histoire » aux éditions Antipodes.

\section{FRANÇOIS VALLOTTON}

François Vallotton est professeur ordinaire d'histoire contemporaine à l'Université de Lausanne. Auteur de nombreuses contributions sur l'histoire culturelle et intellectuelle, il a consacré de 
nombreux ouvrages et articles à l'histoire du livre et de l'édition, à l'histoire de la presse ainsi qu'à l'histoire de la radio et de la télévision, dans une perspective suisse mais aussi transnationale. Il est également membre fondateur du Centre interdisciplinaire des Sciences historiques de la culture (Université de Lausanne). 